\title{
PRIMARY INVESTIGATION ON POTENTIAL INTERACTION OF AN ER: YAG LASER SYSTEM WITH SKIN
}

\author{
Sana M balleg ${ }^{*}$, Iman M Amesawi $^{2}$, and Abtisam A. Alakrout ${ }^{3}$ \\ ${ }^{1,2}$ Department of Physics, Facluty Of Science-El Ajelat. Zawia University \\ ${ }^{3}$ Department of Physics, Faculty of Sciences, Sabratha University \\ ballegsanaa@yahoo.com
}

\begin{abstract}
Lasers are becoming widely used in medicine due to their beneficial effects such as: coagulation properties (less postoperative bleeding). As an alternative, lasers produce invisible infrared light at a wavelength of $2.940 \mathrm{~nm}$ which is ideal for absorption by hydroxylapatite and water. Therefore, they can be used for the treatment of both soft and hard tissues. The aim of this study is to investigate the interaction between the laser systems with power $15 \mathrm{~W}$ and pulse repletion rate up to $1 \mathrm{KHz}$ on chicken skin at various irradiation parameters with distance $10 \mathrm{~cm}$. A beam delivery unit, a focusing camera and a computer controlled stepper unit with sample holder to move the sample while irradiation with different laser parameter were used in this study. After irradiation and to inspect skin geometry and damage light microscopy, image analysis and laser scanning microscopy were used. It was noticed that the surface of the skin was slightly damaged with depth up to $3 \mathrm{~mm}$ and width about $200 \mathrm{~mm}$. This damage disappeared after few minutes of irradiation. In conclusion, the experiment demonstrated that the Er:YAG-laser system is an efficient tool for studying the interaction between the skin and laser in terms of use in treatment of skin problems. The results also show that when the number of pulses increases the depth of the laser becomes more and the damage increases as well. Further research, including controlled clinical and research studies, to investigate the higher efficacy, as well as side effects of laser therapy, is needed.
\end{abstract}

Keywords: Er:YAG laser; chicken skin; interaction tissue.

\section{Introduction}

When the laser was developed in early of 1960s, its great potential for some medical application was noticed. The qualities of laser light make it deliver a big amount of optical energy to small target on or below the surface (Jorgen Serup and G.B.E.Jemec, ( 1995)). The exact details of laser beam effect on tissue and their causing mechanisms are still being elusive (David H.Sliny and Stephenl.( 1993)). For example, the thermal effects of laser beam incident upon the skin can be predicted by knowing the temperature distribution within the skin and the thermal diffusion of the heat from the exposed spot to the surrounding tissue. The result of laser treatment depends on the immediate interaction between the incident light and the tissue 
as well as the subsequent tissue regeneration and remolding (E.V.Ross, J.R.Mckinlay, F.P.Sajben, C.H.Miller, D.J.Barnette (2002)). The interaction between the erbium laser and the tissue is based on the physics of laser such as, the penetration and the absorption of energy of the chronophers that will basically depend on wavelength, which is $2940 \mathrm{~nm}$; the more the duration of the pulse the more penetration will be. The size of the spot determines the penetration and the energy density needed depending on the flounce and the absorption within the target chromospheres which in these systems is water; the percentage of water stratum corneum of the skin is $15 \%$ (Fernando Urdiales Galvez, (2000)). Since the absorption of water in skin is the main factor of interaction between skin and laser, there are three types of laser that can be effectively used for skin tissue. Er:YAG laser with $2940 \mathrm{~nm}$ wavelength has great absorption through water, 10 times greater than $\mathrm{CO} 2$ and lower temperature transmission to the surrounding tissue than $\mathrm{CO} 2$. The low penetration is shown in Figure (1) (J Drugs Dermatol (2007)).

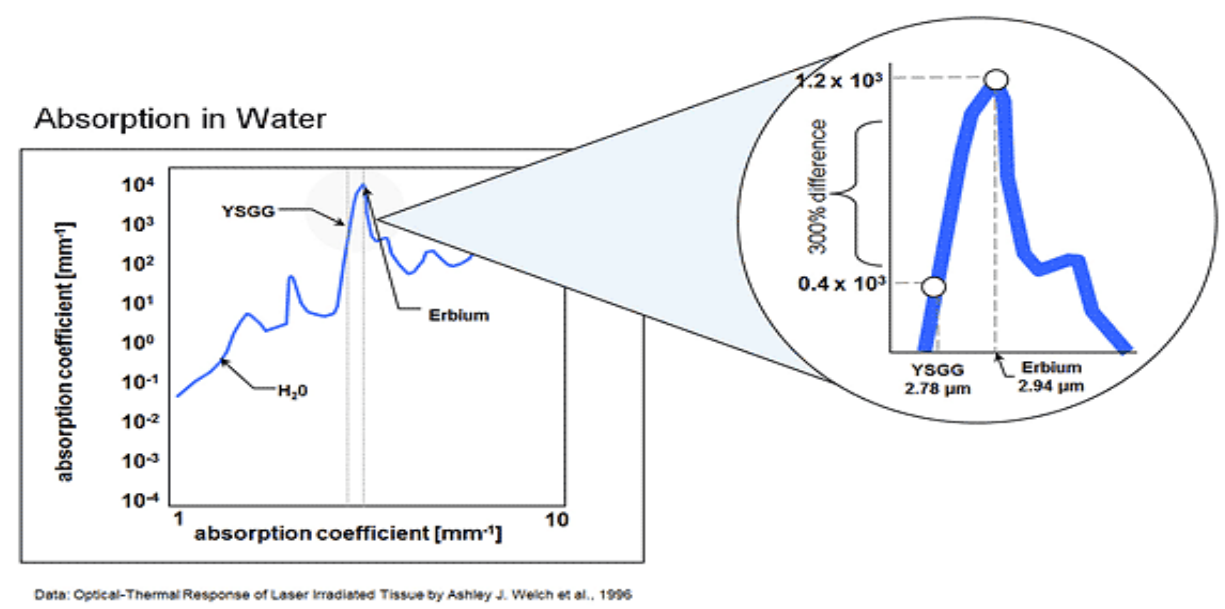

Figure (1): The Effect and Interaction Er:YAG Laser Resurfacing on Skin Flaps.

Initially, the tissue is heated directly upon laser irradiation, within the optical absorption depth. The direct heating is followed by thermal diffusion that indirectly heats the deeper lying tissue. For short pulse, the time span for thermal diffusion is short, and the temperature does not go very deep into the tissue. For longer pulse, the heat has more time to spread deeper in the tissue. The depth of thermally affected skin layer is reduced because the hottest part of tissue which is close to the surface evaporates. (MatjazLuckac, ZdenkoVizintin, Marko Kazic (2008)).

Er:YAG laser radiation of $2.94-\mu \mathrm{m}$ has extremely short penetration depth in water $(\sim 1 \mu \mathrm{m})$ and , hence it allows tissue ablation with a micrometer precision (Kermany O, Lubatschowski H, Asshauer T, (1993), (Visuri SR, Walsh JT, Wigdor HA. (1996)). It has been successfully used 
in dentistry, orthopedics and neurosurgery (Zahn H,Jungnickel V, E, T, Schmid S, Muller G. (1997)). The development of Argon, Neodymium (Nd), YAG and Carbon Dioxide lasers in general areas of surgery led to a gradual introduction of these wavelengths in surgical procedures used in the mouth. These early lasers have a continuous-wave emission mode, which gave rise to potential for conductive heat damage. This was addressed by introducing pulsed-emission lasers, which allowed selective destruction of abnormal or diseased tissue while leaving surrounding normal tissue undisturbed. They were the first lasers to fully exploit this principal of 'selective thermolysis (Zahn H,Jungnickel V, E, T, Schmid S, Muller G. (1997)). This study is to evaluate in a systematic and prospective manner the effect and interaction of Erbuim:YAG laser resurfacing on skin flaps.

\section{Material and Methods}

1. a chicken skin.

2. an Er;YAG laser.

3. a CDC camera.

4. a computer using Image $\mathbf{J}$ software.

\section{Experimental Animals}

Chicken skin was used in this study. This experimental animal was chosen because of its skin structure which is similar to human skin $(9,10)$. We used 4-7 g of chicken samples.

\section{Experimental Flaps}

Ten fresh Skin flaps, including some meat and fats, with 2-4 mm thickness, were taken from each chicken. The flaps were not similar in size and weight. The irradiation modality was randomly assigned for each sample. The samples were weighed

Table (1): experimental groups

\begin{tabular}{|c|c|c|}
\hline Experimental Groups & Samples & Number of Samples \\
\hline Control & Samples Defect & 10 \\
\hline Erbuim:YAG Laser Resurfaced & Samples Immediate from 1-10 Pulses & 100 \\
\hline
\end{tabular}

\section{Experimental Protocol}

In order to capture the effect of laser on skin immediately, the samples were photographed at the time they were irradiated with Erbuim:YAG laser with different pulses. The photographs of skin and section skin were taken at the same distance between the camera and the samples which was about $3 \mathrm{~cm}$. The area and depth of the damage on each sample were measured from the photos. 


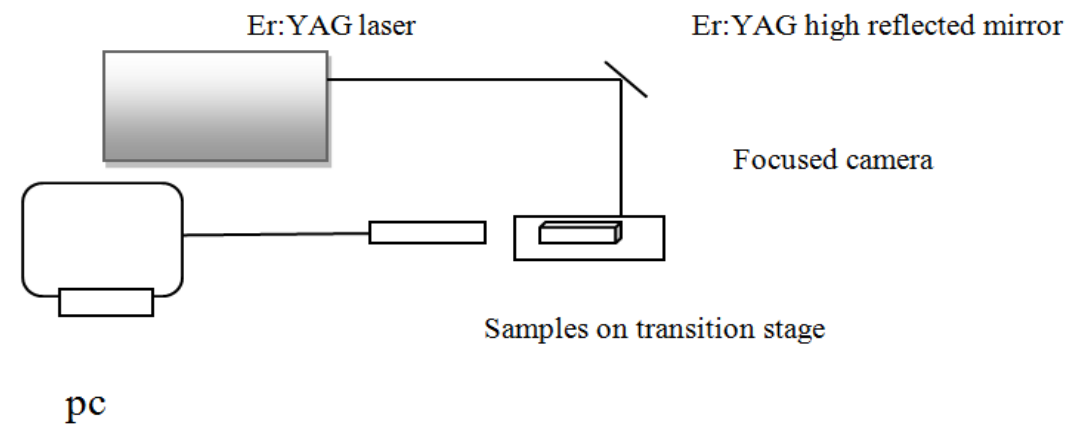

Figure (2): Experimental Setup for Irradiation of the Sample with Focused Camera.

The principle experimental setup is shown in Figure (2). The used Er:YAG laser was with wavelength $2940 \mathrm{~nm}$, pulse duration of $135 \mu \mathrm{s}$. For suitable positioning of the sample an x,y,z transition holder was used to get right position. A computer and a special sample holder controlled the position and movement of the samples and allowed fixing the sample surface in a reproducible z- position. At first the focus and depth were determined by irradiation.

\section{Analysis of the Samples}

During irradiation the interaction through the skin was recorded by camera, after irradiation the photographs were analyzed to measure the damage and depth. From the photos that were captured by camera during the moment of irradiation, it can be clearly seen that the damage on skin travelled through the skin down to the meat; this can be attributed to heat diffusion from the skin to the meat. The damaged area increases by increasing the number of pulses as shown in Figure (3) and Table (2).

Table (2): Shows the Relation Between the Number of Pulses and Depth

\begin{tabular}{|c|c|}
\hline Depth $\mathbf{~ m m}$ & Number of Pulses \\
\hline 39.5 & 1 \\
\hline 68.86 & 2 \\
\hline 70.5 & 3 \\
\hline 73.85 & 4 \\
\hline 73.85 & 5 \\
\hline 75.77 & 6 \\
\hline 74 & 7 \\
\hline 75.2 & 8 \\
\hline 80.9 & 9 \\
\hline 82.34 & 10 \\
\hline
\end{tabular}




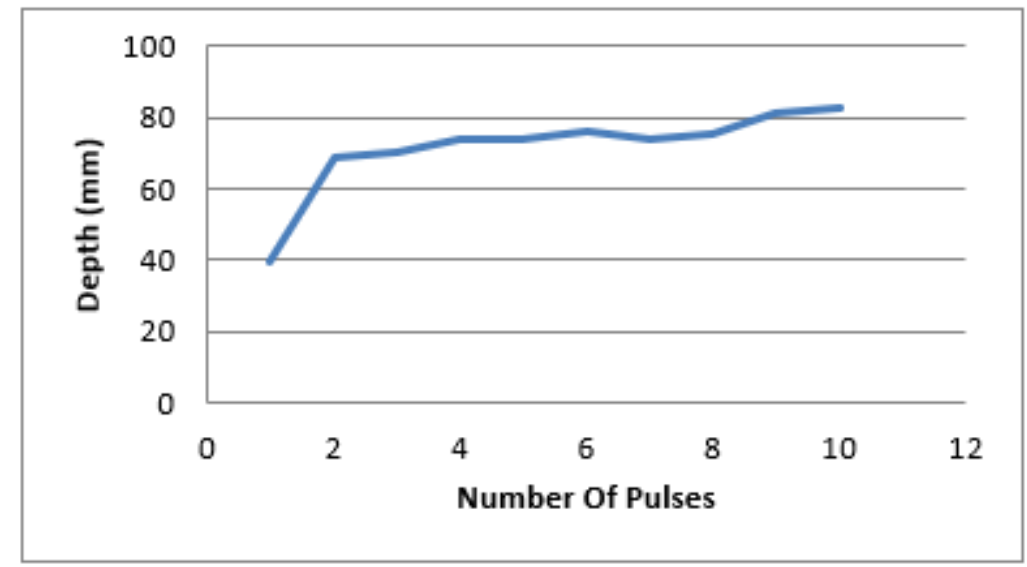

Figure (3): Depth of Er:YAG Laser in Skin Versus Number of Pulses.

When the researchers changed the energy, they noticed that varying energy strength affected the temperature and damage of the skin. Using multi thermal, the temperature before, during and after irradiation was recorded until it reached room temperature again. It can be seen that the damage increased by increasing the energy of laser as shown in the damaged area photos captured by the CCD camera in Figure (4) and in the results analysis in Table (3).

Table (3): The Results Analysis

\begin{tabular}{|c|c|}
\hline Area of Damaged Skin mm & Number of Pulses \\
\hline 5885 & 1 \\
\hline 5930.83 & 2 \\
\hline 5995.1 & 3 \\
\hline 5995.18 & 4 \\
\hline 7532.16 & 5 \\
\hline 7650.08 & 6 \\
\hline 9152.66 & 7 \\
\hline 10411.6 & 8 \\
\hline 10971.9 & 9 \\
\hline 12676.8 & 10 \\
\hline
\end{tabular}




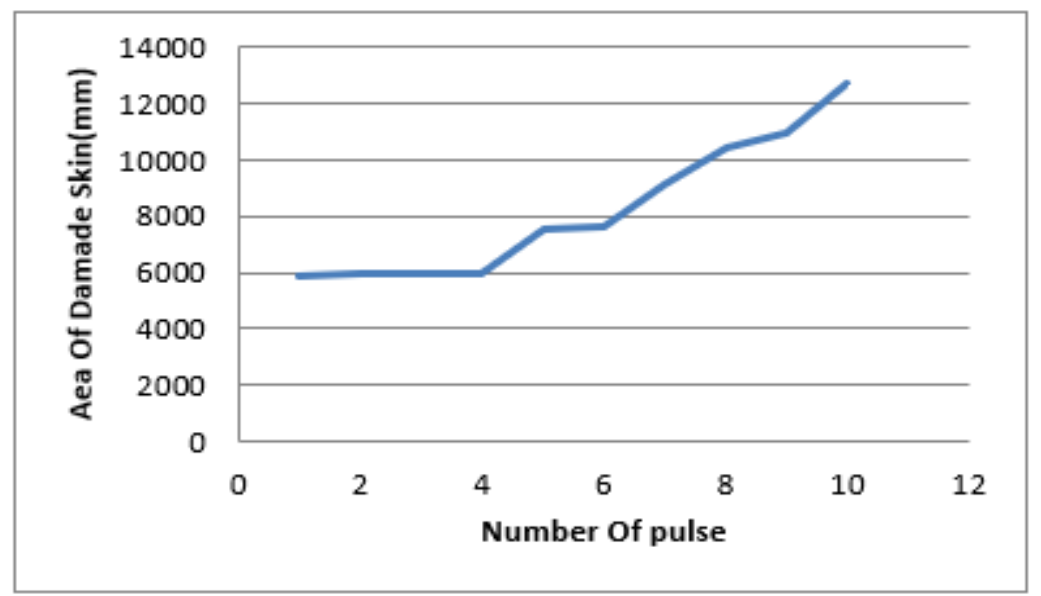

Figure (4): Damage Area of Skin Versus Number of Pulses.

A

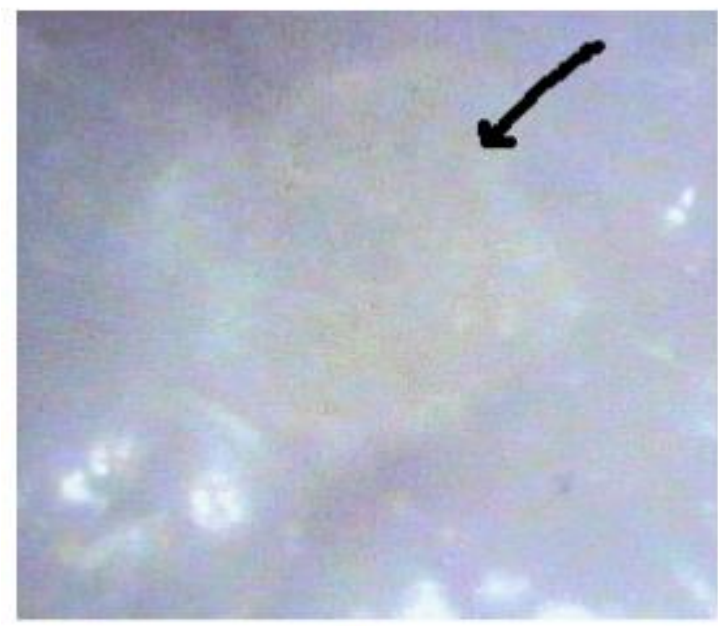

$\mathrm{C}$

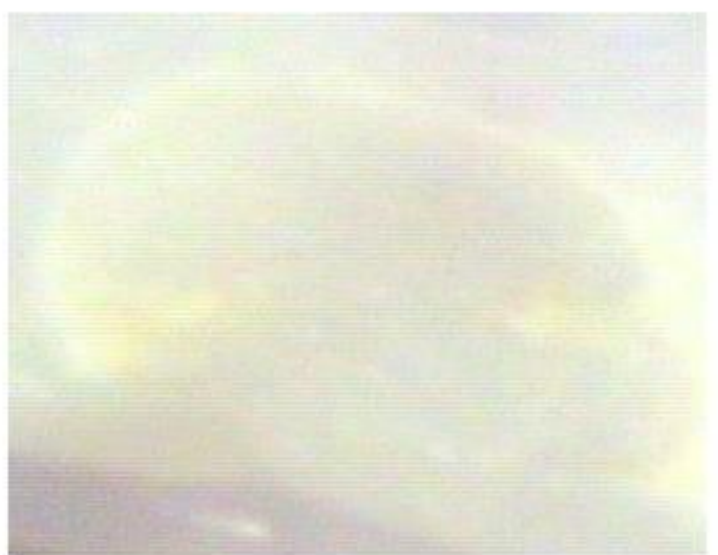

B

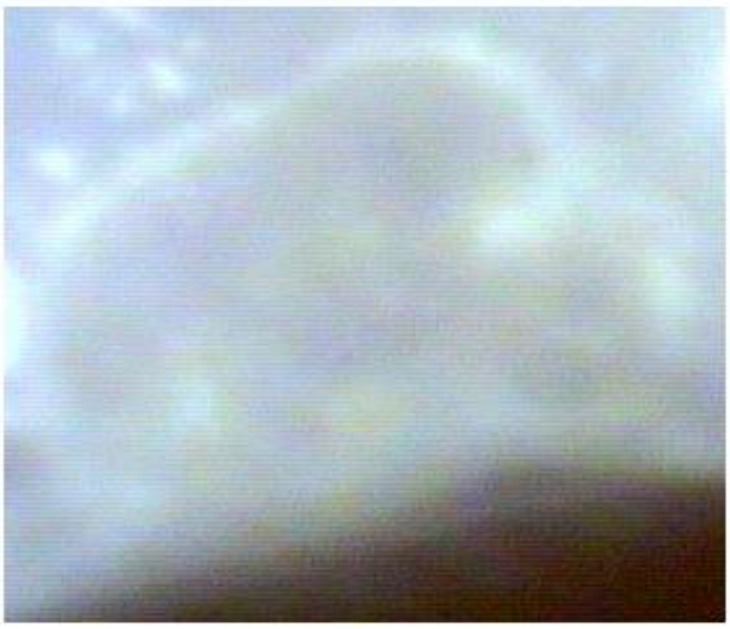

D

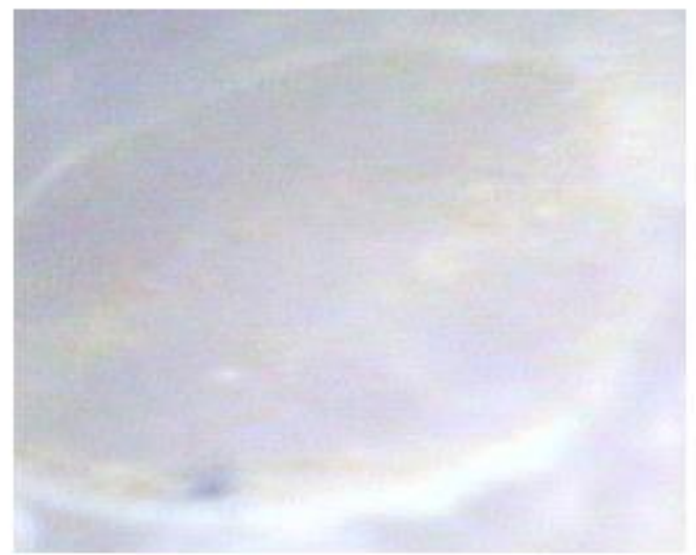

Figure (5): Photos of the Skin Damaged with Different Energy of Er:YAG Laser (A),(B),(C),(D) 89.8mJ,128.5mJ,160.7mJ, 162.5mJ. 


\section{Discussion}

By measuring the weight of samples after and before irradiation, we proved that Er:YAG has a high rate of water absorption. We noticed that there was a loss of weight through the absorption of water by laser; the skin contains $15 \%$ of water. We measured some kind of linear dependency between the number of pulses and depth Figure (3). This can mean that increasing the number of pulses increased the energy and led to more heat diffusion through the skin. Consequently, the damaged area increased as shown in Figure (4). Er:YAG laser light energy must be delivered to the target skin in a temporal pulse of appropriate duration in order to control skin heating and ensure the efficacy, efficiency and safety of the treatments. In case a long laser pulse or continuous irradiation, the heat that is generated by the laser light has sufficient time to diffuse deeper into the tissue from the irradiated surface area. This results in lower ablation efficacy and higher thermal effects inside the skin (Tom Sult M.D., Robin Sult R.N, (2007)). The output of most clinical lasers is Gaussian in a special profile. Therefore, cells directly in the center of the beam are irradiated at a very high fluence, while those on the periphery of the incident beam receive a very low dose. As a result, cells at the beam center may be overstimulated far above the scientifically recommended range of 3-10 $\mathrm{J} / \mathrm{cm}^{2}$ and inhibited while those on the periphery receive insufficient cellular energy to produce any effect. Because of these problems, a hand piece was developed to provide homogeneous irradiation over a $1 \mathrm{~cm}^{2}$ surface and has the same irradiation area (spot size) from contact to $135 \mathrm{~cm}$ of distance from the target tissue (Tom Sult M.D., Robin Sult R.N, (2007)). The optical properties of the skin mimic a turbid medium intermixed with focal discrete visible and infrared light absorbers (blood, melanin, bilirubin, and dry collagen). The thermal or photochemical effects depend on the local energy density at the target. Once the light penetrates the surface, it undergoes a series of absorbing and scattering events. Photons statistically are either scattered or absorbed in a wavelength dependent fashion.1,57 scattering is affected by the shape or size of the particle and the index of refraction mismatch between the particle and medium. For most tissues, for $1>2.5 \mathrm{~mm}$ or less than $250 \mathrm{~nm}$, absorption dominates over scattering. For the remainder of the EM spectrum, scattering is the primary attenuator of light in tissue (KeyvanNouri (2011)). One of the first reports on the use of square shaped pumping for Er:YAG lasers is in ref. (Lukac M, Nemes M, Cencic S, (May 1993)). In another work, the dependence of the input pump energy Eth on the square-shaped pump pulse power was studied experimentally and analytically. In another early study, the influence of square pulse pumping on $\mathrm{Er}(50 \%)$ :YAG laser efficiency was analyzed by numerically solving laser rate equations (JanezDiaci (2012)). The numerical simulations showed that for squareshaped, VSP pumping, the pump energy threshold Eth increased with pump pulse length Tp.

\section{Conclusion}

In conclusion, the present results show the effect of the Erbuim:YAG laser on skin and how interact with tissue. Frequently, several thermal effects are induced in biological tissue, depending on the laser parameters. These effects range from carbonization at the tissue surface 
to hyperthermia a few millimeters inside the tissue. Therefore, careful evaluation of the required laser parameters is essential. Reversible and irreversible tissue damage can be distinguished. Carbonization, vaporization, and coagulation certainly are irreversible processes because they induce an irreparable damage. Depending on the type of tissue and laser parameters, hyperthermia can be either a reversible or an irreversible process. Further research needs to be done to find out the right irradiation parameters and the measurement of tissue temperature.

\section{References}

- David H.Sliny and StephenL. (1993), Trockel.Mediacl Laser and Their Safe Use. SpringerVerlag.

- E.V.Ross,J.R.Mckinlay,F.P.Sajben,C.H.Miller,D.J.Barnette (2002), use of novel erbium laser in Yucatan minipig: study of residual thermal damage, ablation and wound healing as function of pulse duration. Laser sur.Med.30, 93-100.

- Fernando Urdiales Galvez, (2000), fraction rejuvenation of skin with pixel erbium and co2 lasers surg 835-843.

- J Drugs Dermatol (2007), Gold MH. Fractional technology: a review and clinical approaches. 6:849-852.

- JanezDiaci (2012), Comparison of Er:YAG and Er,Cr:YSGG lasers used in dentistry-Journal of the Laser and Health AcademyVol. 2012, No.1.

- Jorgen Serup and G.B.E.Jemec, (1995), Handbook of -Invasive Methods and Skin. CRC Press.

- Kermany O, Lubatschowski H, Asshauer T, (1993) et al. Q-Switched CTE: YAG (2,69 M-m) laser ablation: basic investigations on soft (comeal)and hard (dental) tissues. Lasers Surg Med. 13:537-542.

- KeyvanNouri (2011), Lasers in Dermatology and Medicine- Springer-Verlag London Limited.

- Lukac M, Nemes M, Cencic S, (May 1993), Thermal Load and Efficiency Optimization for a Flashlamp-Pumped Er:YAG laser, Summaries of papers presented at the CLEO in Baltimore, 302-30315.

- MatjazLuckac, ZdenkoVizintin, Marko Kazic (2008), Tom Sult-Novel Fractional Treatment with VSP Erbuim YAG Aesthetic Laser.

- Nagy Abdulsamee (2017), All Tissues Dental Laser Er:YAG laser- Biomed J Sci\& Tech Res Volume 1- Issue 1. 
- Prof Stefano Benedicenti, Prof. a.c Andrea Amaroli (2019), Photobiomodulation with flat top hand piece: from science to the clinic-22-June-2019.

- Tom Sult M.D., Robin Sult R.N, (2007), New Options and Treatment Strategies with the VSP Erbium YAG Aesthetics Lasers- Journal of the Laser and Health Academy Vol. No.1.

- Visuri SR, Walsh JT, Wigdor HA. (1996), Erbium laser ablation of dental hard tissue: effect of water cooling. Lasers Surg Med, 18:294-300.

- Zahn H,Jungnickel V, E, T, Schmid S, Muller G .(1997),.Knochenchirurgie mit dem Er:YAG laser .lasermedizin.13:31-36MatjažLukač Ph.D. 